\title{
Onde se graduam os Médicos de Família e Comunidade do Brasil?
}

\section{Where family and community physicians from Brazil get their medical degree? ¿Dónde se graduaron los médicos de familia y comunidad de Brasil?}

\author{
Thiago Dias Sarti ${ }^{\circledR}$, Leonardo Ferreira Fontenelle ${ }^{2 \oplus}$, Bruno Luciano Carneiro Alves de Oliveira ${ }^{3 \oplus}$, Stephani Vogt Rossi ${ }^{2 \oplus}$, \\ Miguel Henrique Moraes de Oliveira ${ }^{2}$, Ana Paula Santana Coelho Almeida ${ }^{1}$ \\ ${ }^{1}$ Departamento de Medicina Social, Universidade Federal do Espírito Santo, Vitória, Espírito Santo, Brasil \\ ${ }_{2}^{2}$ Faculdade de Medicina, Universidade Vila Velha, Vila Velha, Espírito Santo, Brasil. \\ ${ }^{3}$ Departamento de Medicina, Universidade Federal do Maranhão, Pinheiro, Maranhão, Brasil.
}

\section{Resumo}

Pouco se sabe sobre as características da graduação médica dos Médicos de Família e Comunidade do Brasil. Objetivo: caracterizar as instituições de ensino superior (IES) onde se graduaram os médicos que se especializaram em medicina de família e comunidade no Brasil até 2018. Métodos: estudo descritivo baseado em dados secundários dos currículos Lattes de todos os MFCs que atuam no Brasil, compreendendo informações sobre o local e ano de graduação do médico. Resultados: foram localizados os currículos de $4.065(65,2 \%)$ dos 6.238 MFCs identificados, sendo que 3.889 $(62,3 \%)$ registraram a IES na qual se graduaram em medicina. $3.769(96,9 \%)$ MFCs se formaram em 169 IES brasileiras e $120(3,1 \%)$ se formaram em 48 IES estrangeiras. A maioria dos MFC se graduaram em IES públicas $(n=2.396 ; 63,8 \%)$, localizadas em cidades com mais de 100 mil habitantes ( $n=3.446 ; 96,7 \%$ ) e nas regiões sul e sudeste do país. A região Sudeste concentrou as IES responsáveis pela graduação de mais de $45,0 \%$ dos MFCs. Todos os estados das regiões Nordeste, Centro-Oeste e Norte respondem, cada um, pela graduação de menos de $4,5 \%$ do total de MFCs do Brasil. As IES privadas ganharam maior importância na graduação de MFC a partir de 2008. Conclusão: a maior parte dos MFC que obtiveram seus títulos da especialidade no Brasil foram graduados em medicina no próprio país, em IES públicas localizadas nos grandes centros urbanos das regiões sul e sudeste do país, padrão que se assemelha ao da formação médica em geral.

Palavras-chave: Medicina de Família e Comunidade; Atenção Primária à Saúde; Educação de Graduação em Medicina; Faculdades de Medicina
Como citar: Sarti TD, Fontenelle LF, Oliveira BLCA, Rossi SV, Oliveira MHM, Almeida APSC. Onde se graduam os Médicos de Família e Comunidade do Brasil?. Rev Bras Med Fam Comunidade. 2021;16(43):2626. https://doi.org/10.5712/rbmfc16(43)2626
Autor correspondente:

Thiago Dias Sarti.

E-mail: tdsarti@gmail.com

Fonte de financiamento:

não se aplica.

Parecer CEP:

CAAE 02957118.2.0000.5064.

Procedência:

não encomendado.

Avaliação por pares:

externa.

Recebido em: 07/07/2020.

Aprovado em: 09/12/2020.

Editor Associado:

Leandro David Wenceslau. 


\begin{abstract}
Little is known about the characteristics of medical graduation in Family and Community Medicine in Brazil. Purpose: to characterize the higher education institutions (HEls) where physicians who specialized in family and community medicine in Brazil graduated by 2018 . Methods: A descriptive study based on secondary data from Lattes platform of all family physicians (FP) operating in Brazil, including information about the place and year of physician's graduation. Results: 4,065 (65.2\%) curricula of the 6,238 FPs identified were located. 3,889 (62.3\%) registered the $\mathrm{HEl}$ in which they graduated in medicine. 3,769 (96.9\%) FPs graduated from 169 Brazilian HEls and $120(3.1 \%)$ graduated from 48 foreign HEls. Most FPs graduated from public HEls $(n=2,396 ; 63.8 \%)$, located in cities with more than 100,000 inhabitants $(n=3,446 ; 96.7 \%)$ and in South and Southeast regions of the country. The Southeast region concentrated the HEls responsible for the graduation of more than $45.0 \%$ of the FPs. All states in the Northeast, Midwest and North regions of the country account for less than $4.5 \%$ of Brazil's total FPs. Private HEls have gained greater importance in FP graduation from 2008. Conclusion: Most FPs who obtained their specialty titles in Brazil were graduated in medicine in their own country, in public HEls located in the large urban centers of the South and Southeast regions of the country, a pattern that resembles that of global medical education in Brazil.
\end{abstract}

Keywords: Family Practice; Primary Health Care; Education, Medical, Undergraduate; Schools, Medical.

\title{
Resumen
}

Se sabe poco sobre las características de la graduación médica en Medicina Familiar y Comunitaria en Brasil. Objetivo: caracterizar las instituciones de educación superior (IES) donde los médicos especializados en medicina familiar y comunitaria en Brasil se graduaron hasta 2018. Métodos: estudio descriptivo basado en datos secundarios de los currículos Lattes de todos los MFCs que operan en Brasil, que incluye información sobre el lugar y el año de graduación del médico. Resultados: se ubicaron los planes de estudio de 4,065 (65,2\%) de los 6,238 MFCs identificados. $3,889(62,3 \%)$ registraron la IES en el que se graduaron en medicina. 3,769 (96.9\%) MFCs se graduaron de 169 IES brasileñas y 120 (3.1\%) se graduaron de 48 IES extranjeras. La mayoría de los MFCs se graduaron de IES públicas $(n=2,396 ; 63.8 \%)$, ubicadas en ciudades con más de 100,000 habitantes $(n=3,446 ; 96.7 \%)$ y en las regiones Sur y Sureste del país. La región sudeste concentró las IES responsables de la graduación de más del $45.0 \%$ del MFCs. Todos los estados del Noreste, Medio Oeste y Norte del país representan cada uno menos del $4.5 \%$ del total de MFCs de Brasil. Las IES privadas han ganado mayor importancia en la graduación de MFCs desde 2008. Conclusión: La mayoría de los MFCs que obtuvieron sus títulos de especialidad en Brasil se graduaron en medicina en su propio país, en IES públicas ubicadas en los grandes centros urbanos de las dos regiones Sur y Sureste del país, un patrón que se asemeja al de la educación médica en general.

Palabras clave: Medicina Familiar y Comunitaria; Atención Primaria de Salud; Educación de Pregrado en Medicina; Facultades de Medicina.

\section{Introdução}

Sistemas de saúde são mais efetivos, eficientes e seguros quando há uma rede de serviços de Atenção Primária à Saúde (APS) consolidada, resolutiva, integrada e facilmente acessível ${ }^{1}$. Para tanto, se requer um conjunto de profissionais também adequadamente formados que prestam cuidados abrangentes longitudinais ${ }^{2,3}$. No decorrer das últimas três décadas, o Brasil enfrentou o problema das baixas coberturas de APS induzindo a criação e expansão da Estratégia Saúde da Família (ESF). Contudo, esse esforço ainda não alcançou cobertura universal e permanecem importantes problemas de acessibilidade e qualidade dos cuidados prestados pelas equipes de saúde ${ }^{4,5}$.

Na última década, o país passou a debater e intervir com mais intensidade sobre a questão da disponibilidade e qualificação de profissionais médicos para ocupar os postos de trabalho da APS no Sistema Único de Saúde (SUS) ${ }^{6}$. Neste contexto, ganha importância a discussão sobre o número insuficiente de Médicos de Família e Comunidade (MFC) no país, sendo este um especialista formado a partir de uma matriz de competências especificamente alinhada para o contexto da APS ${ }^{7,8}$. Coloca-se como questão central neste debate a de quais seriam as melhores formas de se formar, qualificar e distribuir os MFCs com vistas a aumentar a qualidade dos serviços e reduzir iniquidades de acesso à atenção à saúde ${ }^{9}$. Observou-se crescente literatura sobre essa temática nos últimos anos, muito devido ao Programa Mais Médicos (PMM) e ao grave problema da alocação e fixação de médicos na APS pública brasileira ${ }^{6}$. Criado em 2013, o PMM é um importante programa governamental de provisão emergencial de médicos para o interior e periferias 
das grandes cidades do país, que buscou também modificar algumas das características da formação médica brasileira, como o número e a distribuição territorial das escolas médicas e as características de seu currículo ${ }^{6,8,9}$.

No entanto, há lacunas de conhecimento sobre a formação dos MFCs que atuam no país, principalmente em relação às características e perfil da sua graduação médica. Pelo conhecimento disponível, não há estudos que analisam o perfil de graduação médica dos médicos que atuam no Brasil e que optaram pela MFC como especialidade, por exemplo. Este tipo de informação é relevante para se entender os fatores que se associam à escolha futura que um estudante de medicina faz por seguir determinada especialidade médica no contexto brasileiro. Sabe-se que esta escolha é complexa e envolve fatores tão diversos como organização social, expectativas profissionais individuais e sociais, desenvolvimento institucional, dentre outros $^{9,10}$. O Brasil promove significativos esforços para induzir a escolha da especialidade médica pelos graduandos em acordo com as necessidades de seu sistema de saúde. Mas, em grande medida, as políticas são desenhadas sem base científica apropriada, o que pode resultar em desperdício de recursos escassos e baixa qualidade das intervenções ${ }^{9,11}$.

Assim, o objetivo deste estudo é caracterizar as instituições de ensino superior (IES) onde se graduaram os médicos que se especializaram em MFC no Brasil desde 1981, quando se iniciou o registro dos MFCs que concluíam a residência médica, até o ano de 2018.

\section{Métodos}

Trata-se de um estudo descritivo baseado em dados secundários oriundos do Sistema de Informações da Comissão Nacional de Residência Médica (SisCNRM), da base de dados de médicos titulados pela Sociedade Brasileira de Medicina de Família e Comunidade (SBMFC/AMB), da Plataforma Lattes e do Cadastro e-Mec de Instituições e Cursos de Educação Superior do Ministério da Educação. Descrevemos com mais detalhes o delineamento e obtenção dos dados em outro trabalho ${ }^{12}$.

Foram identificados todos os MFC do Brasil a partir do cruzamento dos dados extraídos do SisCNRM, dos médicos que concluíram a residência médica (RMMFC) referentes ao período de 1981 a 2018 e da lista de titulados pela SBMFC/AMB (TEMFC) referentes ao período de 2003 a 2018. O currículo dos médicos foi localizado na Plataforma Lattes buscando pelo Cadastro de Pessoa Física (CPF) ou, quando necessário, manualmente pelo nome. Os currículos, quando disponíveis, foram copiados em formato XML (eXtensible Markup Language) em dezembro de 2018, permitindo acesso às variáveis do estudo. Mais detalhes sobre esse processo estão disponíveis em outro artigo '.

Como variáveis do estudo, identificaram-se a forma de obtenção do título de especialista em MFC (residência médica e/ou prova de título pela SBMFC/AMB); nome, localização (região geográfica do país e estado), porte populacional do município-sede e natureza administrativa (pública ou privada) da instituição de ensino superior (IES) na qual o médico cita ter realizado sua graduação médica; e ano de início da graduação em medicina. Optou-se por considerar o ano de início da graduação a partir da constatação de que parte significativa dos médicos com currículo disponível não indicavam o ano de conclusão de sua graduação, sendo que a imputação de 6 anos de formação como regra universal para a amostra provavelmente resultaria em vieses de informação. 
A análise dos dados se baseia em estatística descritiva com apresentação de frequências absolutas e relativas. O banco de dados foi inicialmente construído no programa Excel, com revisão das informações feita por dois pesquisadores de forma independente. Em um segundo momento, os dados foram analisados no pacote estatístico Stata ${ }^{\circledR}$ (versão 14, Stata Corp., College Station, Estados Unidos).

O estudo foi aprovado pelo Comitê de Ética em Pesquisa da Universidade Vila Velha (CAAE 02957118.2.0000.5064), e o acesso aos dados do TEMFC foi autorizado pela SBMFC. Dados pessoalmente identificáveis foram acessados apenas pela equipe, para fins de pesquisa.

\section{Resultados}

Agregando os bancos SisCNRM e SBMFC/AMB e eliminando as duplicidades, foram identificados 6.238 MFCs com RMMFC ( $n=3.958 ; 65,2 \%$ ) e/ou TEMFC ( $n=2.794 ; 44,8 \%$ ) no país até o final de 2018. Desses MFCs, 4.065 (65,2\%) tiveram seu currículo Lattes localizado, e 3.889 (62,3\%) tiveram sua IES de graduação identificada. Dentre os MFCs com IES identificada, 3.769 (96,9\%) se formaram em 169 IES brasileiras diferentes e $120(3,1 \%)$ se formaram em 48 IES estrangeiras. As IES estrangeiras indicadas pelos MFC como seu local de graduação estão localizadas nos seguintes países: Cuba ( $n=46 ; 38,3 \%$ ); Bolívia ( $n=29 ; 24,2 \%)$; Argentina $(n=12 ; 10,0 \%)$; Colômbia $(n=10 ; 8,3 \%)$; Espanha $(n=6 ; 5,0 \%)$; Itália $(n=3 ; 2,5 \%)$; Venezuela $(n=3 ; 2,5 \%)$; Uruguai $(n=2 ; 1,7 \%)$; Canadá $(n=2 ; 1,7 \%)$; Alemanha $(n=1 ; 0,8 \%)$; Holanda $(n=1 ; 0,8 \%)$; Suíça $(n=1 ; 0,8 \%)$; Peru $(n=1 ; 0,8 \%)$; Paraguai $(n=1 ; 0,8 \%)$; e Equador $(n=1 ; 0,8 \%)$; e Portugal $(n=1 ; 0,8 \%)$.

Em relação às IES localizadas no Brasil, a Figura 1 mostra a distribuição de MFCs formados em escolas por estado da federação e região geográfica. A região Sudeste concentrou as IES responsáveis pela graduação médica de mais de $45,0 \%$ dos MFCs, e os estados do Rio Grande do Sul, Minas Gerais, Rio de Janeiro e São Paulo totalizam cada um mais de 400 MFCs graduados em seu território. Todos os estados das regiões Nordeste, Centro-Oeste e Norte respondem, cada um, pela graduação de menos de $5 \%$ do total de MFCs do Brasil, sendo que no estado do Amapá não se graduou nenhum dos MFC do país. A maioria dos MFCs se graduaram em IES públicas ( $n=2.396 ; 63,8 \%$ ), localizadas em cidades com mais de 100 mil habitantes $(n=3.446 ; 96,7 \%)$ e nas regiões Sul e Sudeste do país (Tabela 1). Todas as 10 IES com o maior número absoluto de egressos MFCs (com mais de 100 médicos no período histórico analisado) eram universidades públicas federais, sendo que as principais foram a do Rio Grande do Sul - UFRGS (4,6\%), Minas Gerais - UFMG (4,2\%), Paraná - UFPR (3,0\%), Ceará - UFC (3,0\%), Rio de Janeiro - UFRJ (3,0\%) e de Ciências da Saúde de Porto Alegre - UFCSPA (2,6\%).

AFigura 2, por sua vez, traz os dados de ingresso na graduação médica em uma perspectiva temporal. Observa-se nela que, de 1981 a 2009, as admissões em IES públicas responderam pela maioria dos egressos que se especializaram em MFC, com um aumento expressivo da importância das IES privadas nos anos mais recentes.

\section{Discussão}

O conjunto dos dados, referentes ao longo período analisado, mostrou que a maior parte dos MFCs com RMMFC e/ou TEMFC que obtiveram seus títulos no Brasil foram graduados em medicina no próprio país, em IES públicas localizadas nos grandes centros urbanos das duas regiões mais ao sul do país. 


\begin{tabular}{|c|c|c|}
\hline UF & $n$ & 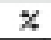 \\
\hline$S P$ & 469 & 24 \\
\hline $\mathrm{PW}$ & 639 & $\nu, 0$ \\
\hline MG & 542 & $x, 4$ \\
\hline ES & 73 & 19 \\
\hline PR & 254 & 7,0 \\
\hline 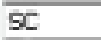 & 181 & 4,3 \\
\hline RS & 606 & $\mathbf{1 8}, 1$ \\
\hline DF & 53 & 17 \\
\hline GD & 27 & 0.7 \\
\hline MT & 63 & 17 \\
\hline MS & 20 & 0.5 \\
\hline TO & 48 & 13 \\
\hline$\overline{P A}$ & 151 & 40 \\
\hline $\mathrm{FR}$ & 8 & 0.2 \\
\hline AMM & 52 & 14 \\
\hline$\overline{\mathrm{AO}}$ & 24 & 06 \\
\hline$A C$ & 16 & 0.4 \\
\hline$\Delta P$ & 0 & 00 \\
\hline$P E$ & s & 25 \\
\hline $\mathrm{PQ}$ & 93 & 25 \\
\hline BA & 59 & 16 \\
\hline ALL & 43 & 11 \\
\hline$P I$ & $v$ & 0,5 \\
\hline$F N$ & 61 & 16 \\
\hline $\mathrm{MA}$ & $x$ & 0.5 \\
\hline CE & 134 & 3.6 \\
\hline$\overline{S E}$ & $\boldsymbol{x}$ & 0.5 \\
\hline
\end{tabular}

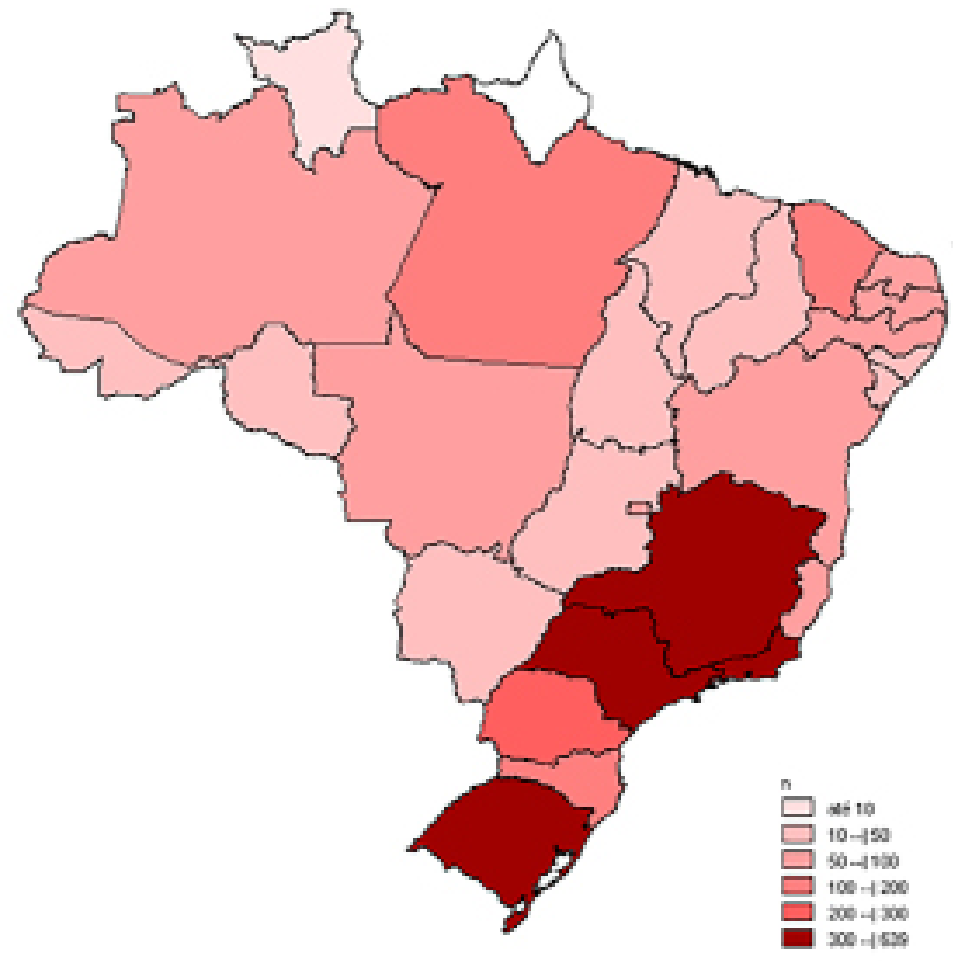

Figura 1. Unidade federativa de realização da graduação médica dos Médicos de Família e Comunidade que obtiveram o título de especialista no país - Brasil, 2018.

Tabela 1. Natureza administrativa, localização e porte populacional do município das instituições de ensino superior de graduação dos Médicos de Família e Comunidade - Brasil, 2018.

\begin{tabular}{|c|c|c|}
\hline Características & $\mathbf{n}$ & $\%$ \\
\hline \multicolumn{3}{|c|}{ Natureza Administrativa $\left(n=3.756^{\star}\right)$} \\
\hline Privada & 1.360 & 36,2 \\
\hline Pública & 2.396 & 63,8 \\
\hline \multicolumn{3}{|l|}{ Região ( $n=3.769$ ) } \\
\hline Sudeste & 1.727 & 45,8 \\
\hline Sul & 1.031 & 27,4 \\
\hline Centro-Oeste & 216 & 5,7 \\
\hline Norte & 252 & 6,7 \\
\hline Nordeste & 543 & 14,4 \\
\hline \multicolumn{3}{|c|}{ Porte populacional do município sede $\left(n=3.563^{\star \star}\right)$} \\
\hline Pequeno (<50 mil hab.) & 41 & 1,2 \\
\hline Médio (50 mil a 100 mil hab.) & 76 & 2,1 \\
\hline Grande (>100 mil hab.) & 3.446 & 96,7 \\
\hline
\end{tabular}

${ }^{*}$ Valores ignorados (missing): $\mathrm{n}=13 .{ }^{* *}$ Valores ignorados $($ missing): $\mathrm{n}=206$.

Este perfil é o mesmo que o encontrado para a formação médica em geral, incluindo o nível de residência médica, e contribui para explicarmos a histórica concentração de médicos nos grandes centros das regiões sul e sudeste e escassez de médicos nas regiões remotas e mais carentes do Brasil ${ }^{13,14}$. 


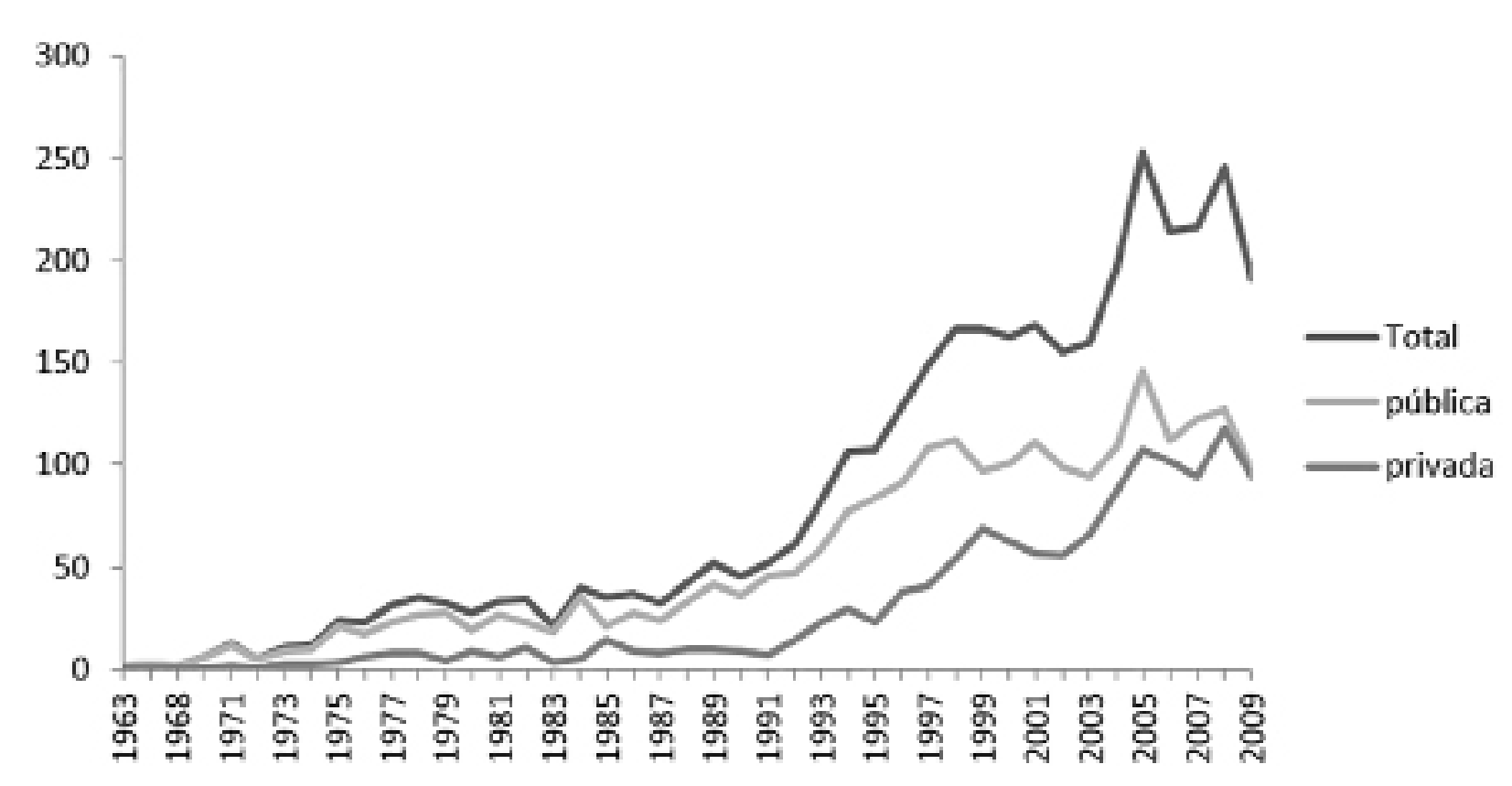

\section{Observação: ano de admissão do profissional na graduação.}

Figura 2 . Natureza administrativa da graduação médica dos Médicos de Família e Comunidade - Brasil, 2018.

Segundo dados do estudo "Demografia Médica 2018"14, entre 1980 e 2017 a população de médicos no país mais que triplicou, passando de 137.347 mil para 451.777 mil, respectivamente, representando aumento de 59,0\% na década de 80,33,0\% na década de $90,24,0 \%$ na de 2000 e 23,0\% entre 2000 e 2017. Esta tendência histórica de crescimento do número de médicos também é observada para a MFC, mas com maior pico de crescimento a partir dos anos 2000. Junto a esse crescimento "tardio" da MFC, observase um significativo aumento do número de MFCs graduados em IES privadas a partir de 2008 (Figura 2).

Este cenário pode ser entendido no contexto das transformações da educação médica e do sistema de saúde do país ocorridas neste período, com maior expressividade da APS no cenário assistencial público e suplementar ${ }^{15}$, das políticas de expansão do ensino médico (com destaque para as escolas privadas) ${ }^{13,16}$ e de reformulação dos currículos acadêmicos com ênfase na integração ensino-serviço-comunidade e inserção precoce na rede de atenção à saúde (em especial, na APS) ${ }^{17}$, além de uma possível influência de políticas de provimento médico (ex. Provab) na APS ${ }^{8}$ e da expansão de programas de residência médica em MFC municipais (ex. Rio de Janeiro) com complementação financeira nos valores das bolsas ${ }^{18,19,20}$. Mesmo não dispondo dos números proporcionais de egressos das IES neste trabalho, tanto globais quanto dos que optaram pela MFC, os resultados nos permitem dizer que tais políticas ainda não foram potentes o suficiente para modificar o padrão centralizado de formação médica no país, incluindo a MFC, em parte porque encontram grandes barreiras de implantação em cenários educacionais e assistenciais precários e de baixa cobertura populacional de APS ${ }^{9,14,21}$. Além disso, uma série de intervenções feitas no país ainda não dispõem de tempo suficiente para mostrar seus resultados de forma plena.

A prevalência de MFCs graduados em IES estrangeiras mostrou-se residual. A dinâmica de participação de médico(a)s formado(a)s em IES estrangeiras no mercado de trabalho brasileiro é pouco 
estudada e provavelmente pouco significativa. Há importantes lacunas de conhecimento sobre os fluxos de brasileiros para IES dos países latino-americanos, como Bolívia e Cuba, e sobre os efeitos disso na prática médica e no mercado de trabalho médico no país. Cuba é sabidamente um país que orienta fortemente seu sistema de saúde e formação médica para a APS, o que pode explicar seu destaque como polo formador de médicos que optam pela MFC no Brasil ${ }^{22}$. Mas dificilmente se pode falar o mesmo da Bolívia, o que provavelmente pode ser explicado pela dinâmica distinta de acesso ao curso médico nestes países por brasileiros, incluindo classe social. Pouco se sabe também dos fatores que influenciam a entrada de médicos estrangeiros no mercado nacional e se os programas de provimento do tipo Programa Mais Médicos modificaram esta realidade.

Os dados deste trabalho ajudam a preencher lacunas de conhecimento das particularidades da formação do MFC no Brasil, mostrando que esta possivelmente se assemelha ao já conhecido padrão de grandes desigualdades na formação e distribuição da força de trabalho médica no país. Por outro lado, em um país profundamente desigual e de grande extensão territorial, os resultados aqui apresentados, mesmo com suas limitações e foco específico, se somam à literatura no sentido de sugerir serem fundamentais políticas indutoras de um perfil médico mais próximo das necessidades de saúde da população brasileira, do modelo prioritário de atenção à saúde (baseado na APS) e de alocação e fixação destes profissionais de uma maneira equânime no território nacional ${ }^{2,9,23,24}$.

A escolha da especialidade médica é um fenômeno complexo envolvendo fatores relacionados à trajetória de vida da pessoa e sua posição na estrutura social, à escola médica e seu currículo, à residência médica e às características do mercado de trabalho em cada especialidade, tornando algumas áreas médicas mais atrativas que outras do ponto de vista de status, salários, rendimentos e disponibilidade de vagas de residência ${ }^{10}$. Da mesma forma, a escolha que o médico faz do local de estabelecimento e manutenção de sua prática é multifatorial, incluindo a atratividade e as oportunidades de trabalho para o profissional e sua família na cidade, potencial de rendimentos financeiros, modalidades de contratação e pagamento de honorários, condições de trabalho e estruturação da rede de serviços de saúde local, reconhecimento, prestígio e uma série de expectativas da pessoa em relação a suas condições de vida ${ }^{9,25}$.

Formular políticas indutoras diante tantos vetores que influenciam e condicionam as escolhas dos médicos para suas carreiras (onde trabalhar e em qual especialidade atuar) é um desafio e nos permite compreender porque o simples aumento do quantitativo de médicos no país pode não resultar em distribuição equitativa destes ${ }^{9,25}$. Os baixíssimos números de egressos das faculdades médicas das Regiões Norte, Nordeste e Centro-Oeste que optam pela MFC sinalizam para problemas de provisão médica em áreas essenciais que impactam negativamente na saúde da população, na medida em que a densidade de médicos na APS parece ter relação direta com maiores níveis de saúde populacional, principalmente quando estes são formados especificamente para este nível de atenção ${ }^{26,27,28}$. Outra possibilidade para essa baixa adesão de graduando médicos à MFC pode decorrer da fragilidade e precariedade dos sistemas de saúde em que esses alunos se formaram que não permitem a eles desenvolver suas habilidades e competências na APS em relação ao sistema hospitalar que tende a ser mais estabelecido nessas regiões ${ }^{21}$.

Os resultados sugerem que a MFC ainda não é escolhida em número desejável como área de atuação profissional pelos graduados em medicina, o que aponta para a manutenção das desigualdades na oferta qualificada do trabalho médico. Assim, frisa-se a importância de estudos que avaliem o impacto e 
efeitos dos programas de provimento médico e reforma do ensino médico implantados nos últimos anos no país como forma de compreender em profundidade a dinâmica das trajetórias profissionais dos médicos, identificando os pontos nevrálgicos que merecem intervenções qualificadas do Estado a fim de atender as necessidades de saúde da população e de organização do sistema de saúde.

Este trabalho apresenta algumas limitações importantes. Um limitador da análise deste dado é a falta de informações proporcionais entre o número de graduandos que se especializaram em MFC e o total de médicos formados nas IES em cada ano. Isto permitiria identificar fatores associados à escolha da MFC como especialidade médica pelos graduados e verificar a tendência histórica de formação de MFCs pelo conjunto das escolas médicas do país. Isto se agrava pelo fato de uma grande parte dos médicos não possuírem currículo Lattes e outra parte não atualizarem seus currículos adequadamente. A existência e atualização do currículo Lattes, por exemplo, pode sofrer influência do local de residência do médico e desfavorecer profissionais de regiões onde seja menos requerido a elaboração deste tipo de curriculum como forma de se apresentar ao mercado de trabalho, como provavelmente seria o caso da Região Norte. Frisa-se a importância da construção de uma base de dados completa, sólida e acessível com relação à formação profissional em saúde, sem a qual sempre existirá dificuldades em se compreender em profundidade a dinâmica do mercado de trabalho na saúde, o que prejudica sobremaneira o planejamento das ações dos órgãos públicos.

\section{Considerações Finais}

Os resultados deste estudo apontaram que a maior parte dos MFC no Brasil foram graduados em medicina no próprio país, em IES públicas localizadas nos grandes centros urbanos das regiões sul e sudeste do país, padrão que se assemelha ao da formação médica em geral. Nos últimos anos, observou-se maior participação das IES privadas na formação de médicos que se especializaram em MFC. Em conjunto, essa realidade sugere heterogeneidade na distribuição dos MFC e sua concentração nas regiões mais ricas, centrais e urbanas do país. Esse padrão de distribuição pode não considerar a diversidade das condições e necessidades de saúde da população e as várias características organizativas do sistema de saúde do Brasil, o que pode manter as iniquidades no acesso à saúde ainda observadas no país

\section{Contribuição dos autores}

a) Thiago Dias Sarti - concepção do projeto do trabalho; construção do banco de dados; interpretação dos dados; redação da versão inicial e final do artigo.

b) Leonardo Ferreira Fontenelle - concepção do projeto do trabalho; construção do banco de dados; interpretação dos dados; revisão crítica do artigo.

c) Bruno Luciano Carneiro Alves de Oliveira - construção do banco de dados; interpretação dos dados; revisão crítica do artigo.

d) Stephani Vogt Rossi - construção do banco de dados; revisão crítica do artigo.

e) Miguel Henrique Moraes de Oliveira - construção do banco de dados; revisão crítica do artigo.

f) Ana Paula Santana Coelho Almeida - construção do banco de dados; análise estatística dos dados; interpretação dos dados; revisão crítica do artigo. 
Todos os autores leram e aprovaram a versão final do artigo submetida, assumindo todas as responsabilidades éticas e legais decorrentes da sua divulgação.

\section{Conflitos de interesse}

Não há conflitos de interesse por parte dos autores em relação ao trabalho.

\section{Bibliografia}

1. Starfield B, Shi L, Macinko J. Contribution of primary care to health systems and health. Milbank Q. 2005; 83(3):457-502. DOI: https:// doi.org/10.1111/j.1468-0009.2005.00409.x

2. Dussault G, Kawar R, Castro Lopes S, Campbell J. Building the primary health care workforce of the 21 st century - Background paper to the Global Conference on Primary Health Care: From Alma-Ata Towards Universal Health Coverage and the Sustainable Development Goals. Geneva: World Health Organization; 2018.

3. Ellner AL, Phillips RS. The coming primary care revolution. J Gen Intern Med. 2017;32(4):380-6. DOI: https://doi.org/10.1007/s11606016-3944-3

4. Malta DC, Santos MAS, Stopa SR, Vieira JEB, Melo EA, Reis AAC. A Cobertura da Estratégia de Saúde da Família (ESF) no Brasil, segundo a Pesquisa Nacional de Saúde, 2013. Ciênc. saúde coletiva. 2016; 21(2):327-38. DOI: https://doi.org/10.1590/141381232015212.23602015

5. Alves MGM, Casotti E, Oliveira LGD, Machado MTC, Almeida PF, Corvino MPF, et al. Fatores condicionantes para o acesso às equipes da Estratégia Saúde da Família no Brasil. Saúde debate. 2014; 38(spe):34-51. DOI: https://doi.org/10.5935/0103-1104.2014S004

6. Mourão Netto JJ, Rodrigues ARM, Aragão OC, Goyanna NF, Cavalcante AES, Vasconcelos MAS, et al. Programa Mais Médicos e suas contribuições para a saúde no Brasil: revisão integrativa. Rev Panam Salud Publica. 2018;42:e2. DOI: https://doi.org/10.26633/ RPSP.2018.2

7. Trindade TG, Batista SR. Medicina de Família e Comunidade: agora mais do que nunca! Ciênc. saúde coletiva. 2016;21(9):2667-2669. DOI: https://doi.org/10.1590/1413-81232015219.18862016

8. Oliveira FP, Araújo CA, Torres OM, Figueiredo AM, Souza PA, Oliveira FA, et al. O Programa Mais Médicos e o reordenamento da formação da residência médica com enfoque na Medicina de Família e Comunidade. Interface (Botucatu). 2019;23(Suppl 1):e180008.

9. Scheffer M. Programa Mais Médicos: em busca de respostas satisfatórias. Interface (Botucatu). 2015;19(54):637-670. DOI: https://doi. org/10.1590/1807-57622015.0245

10. Yang Y, Li J, Wu X, Wang J, Li W, Zhu Y, et al. Factors influencing subspecialty choice among medical students: a systematic review and meta-analysis. BMJ Open. 2019;9:e022097. DOI: https://doi.org/10.1136/bmjopen-2018-022097

11. Scheffer MC, Cassenote AJF, Guilloux AGA, Dal Poz MR. Internal migration of physicians who graduated in Brazil between 1980 and 2014. Hum Resour Health. 2018;16(1):21. DOI: https://doi.org/10.1186/s12960-018-0286-8

12. Fontenelle LF, Rossi SV, Oliveira MHM, Brandão DJ, Sarti TD. Postgraduate education among family and community physicians in Brazil: the Trajetórias MFC project. Fam Med Community Health. 2020;8(3):e000321. DOI: https://doi.org/10.1136/fmch-2020-000321

13. Oliveira BLCA, Lima SF, Pereira MUL, Pereira Júnior GA. Evolução, distribuição e expansão dos cursos de medicina no Brasil (18082018). Trab. educ. saúde. 2019;17(1):e0018317. DOI: https://doi.org/10.1590/1981-7746-sol00183

14. Scheffer M, Cassenote A, Guilloux AGA, Miotto BA, Mainardi GM, et al. Demografia Médica no Brasil 2018. São Paulo: FMUSP, CFM, Cremesp; 2018.

15. Mendonça MHM, Matta GC, Gondim R, Giovanella L, organizadores. Atenção Primária à Saúde no Brasil: conceitos, práticas e pesquisa. Rio de Janeiro: Editora Fiocruz; 2018.

16. Oliveira FP, Pinto HA, Figueiredo AM, Cyrino EG, Oliveira Neto AV, Rocha VXM. Programa Mais Médicos: avaliando a implantação do Eixo Formação de 2013 a 2015. Interface (Botucatu). 2019;23(Suppl 1):e170949.

17. Dias HS, Lima LD, Teixeira M. A trajetória da política nacional de reorientação da formação profissional em saúde no SUS. Ciênc. saúde coletiva. 2013;18(6):1613-1624. DOI: https://doi.org/10.1590/S1413-81232013000600013

18. Justino JLA, Oliver LL, Melo TP. Implantação do Programa de Residência em Medicina de Família e Comunidade da Secretaria Municipal de Saúde do Rio de Janeiro, Brasil. Ciênc. saúde coletiva.2016;21(5):1471-1480.DOI: https://doi.org/10.1590/1413-81232015215.04342016 
19. Storti MMT, Oliveira FP, Xavier AL. A expansão de vagas de residência de Medicina de Família e Comunidade por municípios e o Programa Mais Médicos. Interface (Botucatu). 2017;21(Suppl 1):1301-1314. DOI: https://doi.org/10.1590/1807-57622016.0511

20. Simas KBF, Gomes AP, Simões PP, Augusto DK, Siqueira-Batista R. A residência de Medicina de Família e Comunidade no Brasil: breve recorte histórico. Rev Bras Med Fam Comunidade. 2018;13(40):1-13. DOI: https://doi.org/10.5712/rbmfc13(40)1687

21. Maciel Filho R. Estratégias para a distribuição e fixação de médicos em sistemas nacionais de saúde: o caso brasileiro [tese]. Rio de Janeiro (RJ): Instituto de Medicina Social da Universidade do Estado do Rio de Janeiro; 2007.

22. González Cárdenas LT, Cuesta Mejías L, Pérez Perea L, Presno Labrador MC, Fernández Díaz IE, Pérez Díaz TC, et al. El Programa del médico y enfermera de la familia: desarrollo del modelo de atención médica en Cuba. Rev Panam Salud Publica. 2018;42:e31. DOI: https://doi.org/10.26633/RPSP.2018.31

23. Arantes LJ, Shimizu HE, Merchán-Hamann E. Contribuições e desafios da Estratégia Saúde da Família na Atenção Primária à Saúde no Brasil: revisão da literatura. Ciênc. saúde coletiva. 2016;21(5):1499-1510. DOI: https://doi.org/10.1590/1413-81232015215.19602015

24. Coelho GC, Antunes, VH, Oliveira A. A prática da Medicina de Família e Comunidade no Brasil: contexto e perspectivas. Cadernos Saúde Pública. 2019;35(1):e00170917. DOI: https://doi.org/10.1590/0102-311x00170917

25. Ono T, Schoenstein M, Buchan J. Geographic imbalances in doctor supply and policy responses. Paris: OECD Publishing; 2014. (OECD Health Working Papers 69).

26. Russo LX, Scott A, Sivey P, Dias J. Primary care physicians and infant mortality: evidence from Brazil. PLoS One. 2019;14(5):e0217614. DOI: https://doi.org/10.1371/journal.pone.0217614

27. Basu S, Berkowitz SA, Phillips RL, Bitton A, Landon BE, Phillips RS. Association of Primary Care Physician Supply With Population Mortality in the United States, 2005-2015. JAMA Intern Med. 2019;179(4):506-514. DOI: https://doi.org/10.1001/jamainternmed.2018.7624

28. Vallejo-Torres L, Morris S. Primary care supply and quality of care in England. Eur J Health Econ. 2018;19(4):499-519. DOI: https://doi. org/10.1007/s10198-017-0898-2 